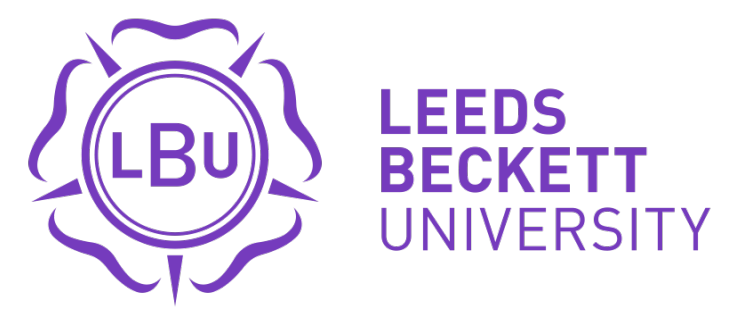

Citation:

Chang, V and Wills, G and Walters, RJ (2011) Towards business integration as a service 2.0 (BlaaS 2.0). In: Proceedings - 2011 8th IEEE International Conference on e-Business Engineering, ICEBE 2011. UNSPECIFIED, 341 - 346. ISBN 9780769545189 DOI: https://doi.org/10.1109/ICEBE.2011.66

Link to Leeds Beckett Repository record:

https://eprints.leedsbeckett.ac.uk/id/eprint/628/

Document Version:

Book Section (Accepted Version)

The aim of the Leeds Beckett Repository is to provide open access to our research, as required by funder policies and permitted by publishers and copyright law.

The Leeds Beckett repository holds a wide range of publications, each of which has been checked for copyright and the relevant embargo period has been applied by the Research Services team.

We operate on a standard take-down policy. If you are the author or publisher of an output and you would like it removed from the repository, please contact us and we will investigate on a case-by-case basis.

Each thesis in the repository has been cleared where necessary by the author for third party copyright. If you would like a thesis to be removed from the repository or believe there is an issue with copyright, please contact us on openaccess@leedsbeckett.ac.uk and we will investigate on a case-by-case basis. 


\title{
Towards Business Integration as a Service 2.0 (BIaaS 2.0)
}

\author{
Victor Chang $^{1,2}$, Gary Wills ${ }^{1}$, Robert John Walters ${ }^{1}$ \\ 1. School of Electronics and Computer Science, University of Southampton, Southampton, UK. \\ 2. Business School, University of Greenwich, London, UK. \\ vic1e09@ecs.soton.ac.uk
}

\begin{abstract}
Cloud Computing Business Framework (CCBF) is a framework for designing and implementation of Could Computing solutions. This proposal focuses on how CCBF can help to address linkage in Cloud Computing implementations. This leads to the development of Business Integration as a Service 1.0 (BIaaS 1.0) allowing different services, roles and functionalities to work together in a linkage-oriented framework where the outcome of one service can be input to another, without the need to translate between domains or languages. BIaaS 2.0 aims to allow automation, enhanced security, advanced risk modelling and improved collaboration between processes in BIaaS 1.0. The benefits from adopting BIaaS 1.0 and developing BIaaS 2.0 are illustrated using a case study from the University of Southampton and several collaborators including IBM US. BIaaS 2.0 can work with mainstream technologies such as scientific workflows, and the proposal and demonstration of BIaaS 2.0 will be aimed to certainly benefit industry and academia.
\end{abstract}

Keywords: Cloud Computing Business Framework (CCBF); Linkage; Business Integration as a Service (BIaaS); Linkage and BIaaS Case Studies; and BIaaS 2.0.

\section{Introduction}

Cloud Computing has transformed the way many organisations work and has offered added values for operation management and service computing [1, 3, 4, 9]. As more organisations adopt Cloud, technical and business challenges emerge. In particular there is a need for a standard, or framework to manage both operation management and IT services. To address increasing requirements in organisational Cloud adoption, a structured framework to provide business needs, recommend the best practices and which can be adapted to different domains and platforms is necessary. The proposed framework is called the Cloud Computing Business Framework (CCBF). It is designed to help businesses to maximise added values offered by Cloud Computing, and deliver solutions, recommendations and case studies to businesses. The $\mathrm{CCBF}$ is proposed to deal with four research areas:

- Classification: Identifying the right strategies and business cases for each type of business model.

- (Organisational) Sustainability: Providing a structured framework to measure cloud business performance.

- Portability: Supporting migration of applications and services to clouds and between clouds (of all types).

- Linkage: Understanding and supporting relationships between alternative cloud methodologies, Business Models like IaaS, PaaS and SaaS.

This proposal focuses on Linkage, a new concept; Business Integration as a Service (BIaaS) and case studies confirming benefits for adoption organisations.

\section{Overview of Linkage}

Effective linkage must have the following characteristics [9]:

- Easy to follow.
- Support for review of Cloud business performance at any time.

- Dynamic, versatile and adaptable characteristics permitting translation between domains, such as IT and business, and ability to fit with any type of cloud businesses and technologies at any stage of a project.

- Include core elements for success.

- Characteristics inherited from SOA (as proposed by Papazoglou and Georgakopoulos [17].

Risk Assessment Framework was first introduced by Li [15] to help organisations to identify their business processes and priorities, and all of these can be mapped together. Key benefits include identifying relationships, the best routes between different processes, and risk analysis.

\subsection{How Linkage leads to Business Integration as a} Service (BIaaS)

The Hexagon Model [7, 11] is used as a link between differing methods and projects. There is an obvious benefit: performance presented in the Hexagon Model need not reveal confidential data. This allows performance reviews with confidentiality. A limitation with the Hexagon Model is linkage can take place within the same process or same service. If there are different Cloud projects in different organisations, the Hexagon Model can still be used, but is applicable to each project, but not interactions between different Cloud projects. Hence, alternative methods such as business process or business integration need to be considered and adopted. Linkage via business process allows different activities, roles, and locations within a project which are able to work and complete together. This can break away from domain-specific activities, so that Cloud services in different domains can interact with one another. 


\subsection{Linkage comparisons: BIaaS vs. Supply Chain}

Rungtusanatham et al. [18] introduced the concept of linkages for supply chain, and they define it as "explicit and/or implicit connections that a firm creates with critical entities of its supply chain in order to manage the flow and/or quality of inputs from suppliers into the firm and of outputs from the firm to customers." There is another type of information-based linkage that can improve the visibility of customers' and suppliers' operational activities [12]. Barratt and Barratt [2] present their external and internal supply chain linkages and use a Coffee case study to demonstrate linkages in relationship and business activities between different roles and companies. Although they show a workflow diagram, data analysis and three propositions, their presentation is still a conceptual framework without any implementations or services in place.

Our concept of linkage is encapsulated in BIaaS, which allows different business processes and activities to be integrated and executed on a central or single linkage framework. Results from each process can be independent and can be passed to the next, without the need for translation, massive computation or workflows (at least once) each time. BIaaS linkage has a higher level of influence and impact factor than supply chain linkage alone.

\section{What is BIaaS 1.0?}

Our current work is defined as BIaaS 1.0, which provides linkage between different types of services, and this offers efficiency improvement and time reduction in business processes. BIaaS 1.0 can be an independent solution, or jointly work with ERP and CRM. All different services in BIaaS 1.0 can work within the same framework without barriers in communications or the need to translate between technologies (such as from BPEL to BPMN). Figure 1 show BIaaS 1.0 based on the integration of different techniques, tools and platform. Firstly, it identifies the right business model. Based on the first-level analysis, the result is passed onto either or both of second and third layers of analysis. The second level of analysis focuses on Sustainability Modelling, which is based on Nobel-prized Capital Asset Pricing Model [19] to compute the Cloud business performance. The result is then converted into 3D Visualisation to present the ROI. The third layer of analysis, which focuses on portability, allows different services to move and work on different Clouds in a way transparent to users. It can also demonstrate Risk Controls and Management. The fourth layer of analysis sums up the project review and recommends the best practices for businesses. It is possible to focus on one particular layer of analysis as an independent project, or a combination of selective layers of analysis as a collaborative project.

\subsection{BIaaS versus BPaaS}

Similarly, the results can be for stand alone projects, or collaborative projects. If this is a collaborative project, then results can be passed onto the final stage; the CCBF review. This has similar and comparable outcomes to the ERP and/or CRM. BIaaS is different from Business Process as a Service (BPaaS), which focuses on using BPEL and/or BPMN to present business processes and how they are linked all together. BPaaS works if all processes are within the same department or same domain for the research area. Workflows can represent business entities and identify relationships between each process, and then link all of them in BPMN or BPEL to make this either into an automated process or a standard process used in the organisation. BIaaS has more to offer than BPaaS. Within work for each research area, it already has a series of activities to connect and collaborate between one another.

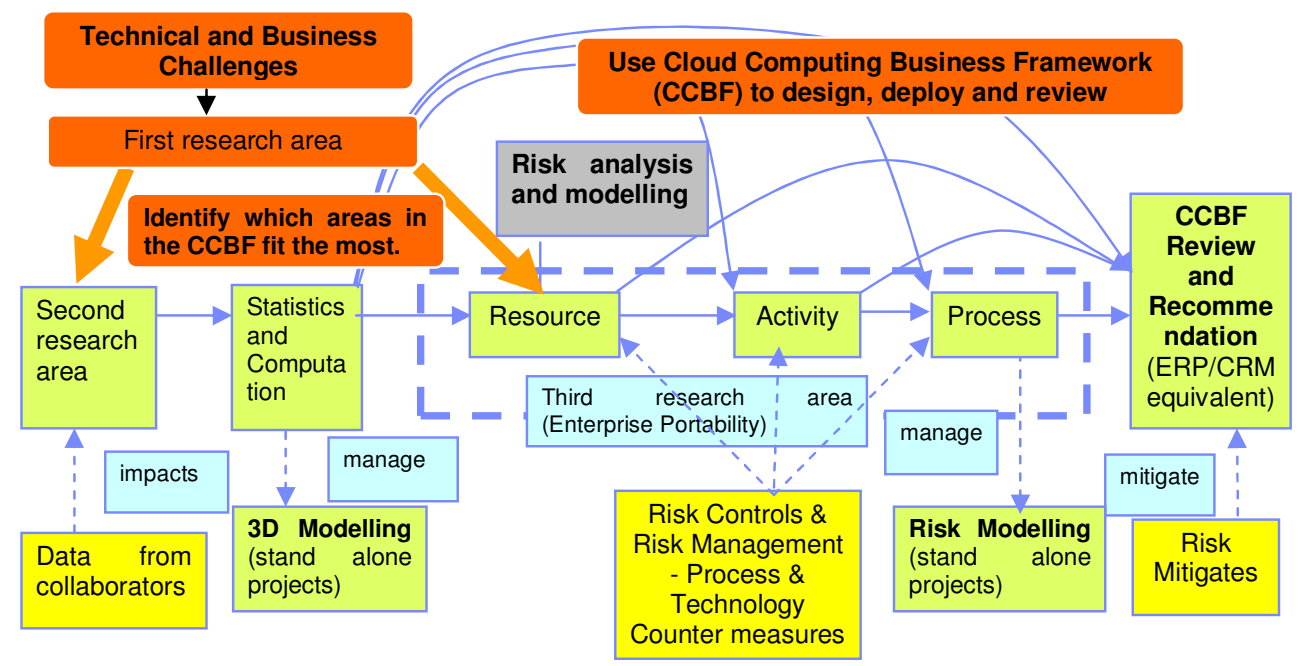

Figure 1: A generic Business Integration as a Service (BIaaS) that the University of Southampton adopts 


\subsection{Advantages of adopting BIaaS 1.0}

Referring to Figure 1, activities in Organisational Sustainability can be considered as BPaaS (not done via BPEL or BPMN). But the challenge is that different business processes in different domains, or in different contexts, need to be able to connect and collaborate. This does not require any translation or schema related interpretation for communication. Linkage is open, and has freedom to link to the respective processes within the framework. Therefore, BIaaS is made available via linkage, which integrates Business Models and IT Services (IaaS, PaaS and SaaS) for service delivery. The CCBF can offer services and connect all services, components, roles and functionalities together. This saves businesses time and resources for analysis, and allows them to compute complex models while having easy to use concepts and features.

\subsection{Desirable Features for the next level, BIaaS 2.0}

BIaaS 1.0 is made up with different technologies to allow different processes to work together. Researchers need to be well trained in different areas to make BIaaS 1.0 happen. Occasionally manual extraction of data and computation is required. Automation is under development.

\section{BIaaS 2.0 Proposal}

BIaaS allows different services, roles, processes and functionalities to work together. Figure 1 shows the first generic model of BIaaS which has been adopted by the University of Southampton (and others) A common challenge is each collaborator has their own agenda, focus and technical preference in their Cloud adoption. BIaaS 1.0 can help organisations reach their goals, certain levels of manual computation and analysis are still required. Full automation allows different processes to be completed electronically. Desirable additional features include full automation, enhanced security, advanced risk modelling and improved collaboration between processes. This can be achieved using Scientific Workflows, because they can present different processes, and improve sharing, collaboration and research analysis amongst research community [13]. Chang et al. [9] also demonstrate BIaaS conceptual framework in Scientific Workflow focusing on MyExperiment (an e-Science platform to share and analyse data), and they present how their work can help to achieve the following $[9,13]$ :

- Understand how developers, users, reviewers and musicians use MyExperiment for digital research and activities, and to suggest any improvements for BIaaS.

- Establish case studies based on users' success stories and to dissimilate knowledge in highly-rated conferences and journals.

Both examples confirm possibilities to exploit Scientific Workflow in BIaaS 2.0, in particular proposing and demonstrating this unique concept that can be applicable to different domains, sectors and areas of specialisation.

\section{BIaaS Case Study at the University of Southampton: Working towards BIaaS 2.0}

The University of Southampton has adopted private cloud initiatives and there are several projects on campus. The School of Electronics and Computer Science at Southampton University (ECS) began to migrate physical servers into virtual servers in 2008, completing the process in December 2009 and then provided services from early 2010. There are two project focuses. One focus is technical, with an emphasis on efficiency improvements. The second focus is cost-saving, and investigates the extent of costsaving Cloud Computing can offer. Meanwhile, Information System Services (ISS) has also consolidated a considerable amount of computing resources, creating an equivalent private cloud pool for the remainder of the University. Their focus has been on confidence and satisfaction for users, gauged from their own analysis and feedback from all students. Most of these projects started in 2009 and completed in 2011.

How the University has adopted linkage and BIaaS is as follows. Firstly, their business models are identified as "In House Private Clouds", "One-Stop Resources" and "Government Funding" based on proposals from Chang et al. [6,7]. These projects are for private clouds, and aimed to improve efficiency and a one-stop service point for staff and students. Following this, ECS has worked closely with us and provided the data, since they are keen to identify the extent of cost-saving that Cloud can offer. Referring to Figure 1, this is the work for second research area, which uses Organisational Sustainability Modelling (OSM), a method to validate cloud business performance.

\subsection{Data Measurement and computation}

OSM is based on the extended Capital Asset Pricing Model (CAPM), which is the analysis of return and risks for organisations or projects in summary. This approach requires organisational metrics and/or detailed interviews. Some firms find it difficult to quantify risk, or risk-free rate. Risk-free rate is the minimum operational costs in cost-saving.

The data collected covers November 2007 to July 2010. CAPM can be modelled by statistical languages, of which SAS is more suitable than others since it can compute more in-depth analysis [7]. SAS code is written to predict the Risk Premiums of an organisation, such as ECS versus the Market (expected values). The data is carefully calculated and examined with data consistency and coding algorithms. Thirty two months of in-depth data represent sustainability from the initial phase to establishment. The SAS program for the CAPM is coded to plot required data using suitable regression methods.

The risk-free rate in this case study means the minimum operational costs in staffing and IT resources. ECS confirms their risk-free rate is reliable, and thus the risk premium is the difference between the expected values and risk-free rate. Apart from OSM, forecasting is an 
important aspect to predict how a cloud business or strategy will perform based on the existing data provided. This is similar to financial markets where forecasting is based on previous data. The difference is that the software market is less volatile than financial markets in which there is greater risk taking. Forecasting is part of OSM to help organisations predict their likely business performance $[10,11]$ and works well in parallel with similar methods.

\subsection{D Visualisation for ECS Cost-saving Model}

Further statistical analysis can be computed. However, this often requires those with relevant training to perform such tasks. Our major contribution in this project is to present complex statistical analysis using 3D Visualisation, so that no data can be missed for analysis, and also those without advanced statistical backgrounds can understand. This is useful for many decision-makers and directors who need to know business analytic results quickly but do not wish spend too much time to understand them.

X-axis: Expected return of cost-saving $(22.5 \%-26 \%)$

y-axis: Actual return of cost-saving $(21.0 \%-22.0 \%)$

z-axis: Risk-free rate $(4.0 \%-4.8 \%)$

Figure 2: 3D visualisation for ECS Cost-saving

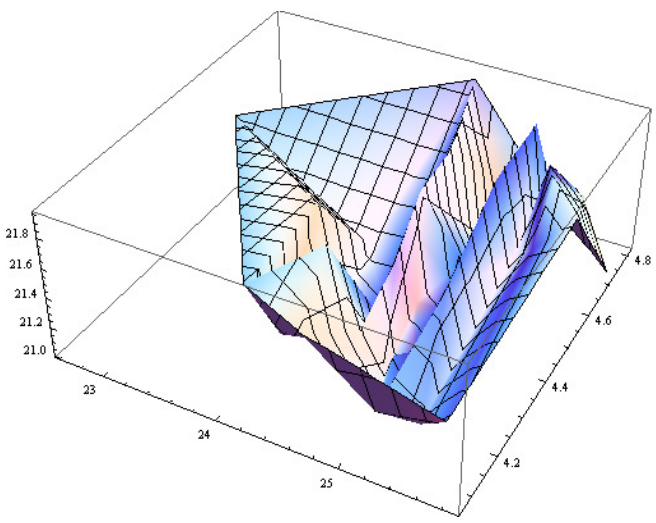

Data is computed in Mathematica and the 3D visualisation models are presented in Figure 2, which is the default 3D model that indicates a high return of cost- saving between $21 \%$ and $22 \%$ on the y-axis, which is significant reduction in operational costs. It also shows the expected cost-saving between 22 and $26 \%$ on $\mathrm{x}$-axis. The $\mathrm{z}$-axis presents risk-free rate (4.0$5.0 \%$ ), which means minimum expenses to keep operation running (including staffing costs). This percentage range can guarantee cost-savings.

With Cloud Computing, statistics can analyse the costsaving from consumption and resources required. But the 3D calculation takes hidden areas such as staffing costs into consideration, which means fewer people are required to do the same amount of work. Similarly, Buyya et al. [5] and Pajorova and Hluchy [16] use 3D Visualisation to present Cloud Computing analysis and challenges. Referring to Figure 1, work from second research area in Section 5.2 is passed to the third, and this process focuses on risk analysis of adopting such approach in the following section.

\subsection{Risk Analysis in BIaaS}

Chang et al. [8] describe financial models they use for risk and pricing analysis, in which they have adopted Monte Carlo Methods (MCM) for advanced risk calculations and Black Scholes Model (BSM) for 3D risk modelling. In this case, the ECS cost-saving is used for risk modelling, where the Least Square Methods (LSM) can be used to compute up to 100,000 simulations in one go to ensure a high level of accuracy. Chang et al. [8] also demonstrate 100,000 simulations can be completed in one go for up to 25 seconds as the maximum time required. This ensures speed and performance are acquired via Cloud computation. To perform risk modelling, American and European options are used, as both models are popular choices within MCM for financial risk analysis. MATLAB (primary language) and $\mathrm{C \#}$ code is written to facilitate a large number of simulations. At the end of computation, it provides the following results.

MCAmericanPrice $=4.9421$

MCEuropeanPrice $=4.3168$

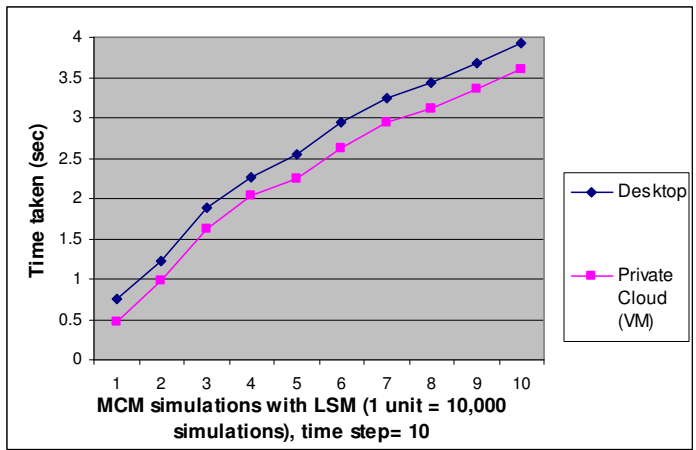

Figure 3: Time taken in MCM simulations

Figure 3 shows the time taken for MCM simulations with time step equal to 10 . For 10,000 simulations, the quickest execution time is 0.5 seconds. Private cloud has a faster execution due to its better hardware capabilities. The key for achieving a fast execution and accuracy still relies on the application, where we have a good QA process to ensure high quality of applications. For 100,000 simulations, maximum time required is less than 4 seconds. Cloud Computing offers fast execution and accuracy due to its computational capabilities. Results from 100,000 simulations are more accurate than smaller numbers of simulations on desktop, and that is a key advantage from Cloud Computing to Operation Management. Both results are useful for decision-makers in ECS to know the impacts of cost-saving. The calculated risk is between $4.3168 \%$ and $4.9421 \%$ in terms of rate. This is likely due to the surge of electricity and operational costs, and such risk rate is under controls most of times.

\subsection{The Outcome of BIaaS 1.0/2.0}

Referring back to Figure 1, when work for Portability has been completed, and the result is passed on to the CCBF Review. This allows the University policy makers to decide the best use of Cloud Computing and its impacts for Operations Management. They can 
understand what is the best business model and operational model for university private cloud, the extent of the cost-saving involved, and analyse the exact risk using a private cloud can offer, plus whether all of these operational and risk events are under control. The entire analysis takes a short time. Unlike some UK government funded projects (their identities cannot be revealed), they take years to build similar systems and they fail to deliver on time, even after receiving further funding. Our linkage approach to integrate different business processes and activities has the capabilities to deliver multiple projects and to provide additional added values. By delivering projects on time, it saves costs in maintenance and future development. MyExperiment is the platform to provide the use of workflow, and is used to help developing the concept and implementation of BIaaS 2.0

\subsection{BIaaS 2.0 Workflow}

BIaaS 2.0 can be demonstrated as a workflow application. A case study is illustrated to present risks in business processes and help making the right business decision. This includes Risk Tolerance, which is commonly associated with the industry framework and business processes and have to be established top down. Chang et al [8] demonstrate a workflow example.

\section{Collaboration with IBM US in BIaaS development}

IBM US, Commonwealth Bank Australia (CBA) and the University of Southampton have worked together for Financial Software as a Service (FSaaS), which also demonstrates BIaaS 1.0 in how risking modelling, risk analysis and security can be integrated and performed for better tasks [8]. These examples include risk modelling, 3D risk visualisation and the use of IBM Fine Grain Security Framework [8, 14].

A major contribution from Southampton University is the use of Monte Carlo Methods (MATLAB) for pricing and Black Scholes Model (Mathematica) for risk analysis. This cloud platform offers calculation for risk modelling, fraud detection, pricing analysis and a critical analysis with warning over risk-taking. It reports back to participating banks and bankers about their calculations, and provides useful feedback for their potential investment. This BIaaS conceptual platform is a working example in BIaaS 1.0.

The CCBF and the IBM Fined Grained Security Framework (IFGSF) will work together forming a hybrid solution to address risk, security and continuous assurance in organisational Cloud adoption. Currently this is at a conceptual framework stage with the following proposal:

- Transparency and privacy - IFGSF can advice users of technologies, techniques and best practices to enforce security, control and monitoring.

- Compliance and trans-border information - $\mathrm{CCBF}$ and IFGSF will work together to fulfil different legislation and data protection laws in the US, UK and EU.
- Certification and user support - IBM has provided relevant Cloud certifications, and CCBF has been adopted in several organisations that have excellent user support and case studies.

Both CCBF and IFGSF will improve on BIaaS in terms of providing advice, consultancy, implementation, and use cases.

\section{The development of BIaaS 1.0 and 2.0 in other organisations}

BIaaS 1.0 and 2.0 from the CCBF have helped several Universities in their design, deployment and migration to Cloud services. Automation, security and collaboration have been added and improved. The examples can be summed up as follows:

- King's College of London (KCL) and Guy's and St Thomas' NHS Trusts have developed Cloud Storage based on IaaS and PaaS solutions. Services are in place to help researchers in backup, automation and data integration. This allows data and backup services to be fully integrated.

- MyExperiment, an e-Science platform developed by the Universities of Manchester and Oxford, has used BIaaS (part of CCBF) to demonstrate how different activities in analysing, processing and sharing digital music can be jointly used.

- The University of Greenwich presents three case studies in the development and migration of Sharepoint, Media Server and Supply Chain private cloud. The Sharepoint project offers three different types of workflow:

1. Examination Papers Workflow: This allows course leaders, moderator, Head of each group, examination officer and external examiners to work together in a sequence of events, including the review process and approval process related to development of examination paper. All these processes are automated.

2. Plagiarism Workflow: When plagiarism is detected by staff using TurnitIn [20], it informs the quality team to start with a sequence of events, such as arranging interviews with students, academic staff and administrators. The interview panel makes the decision, which will influence how markers and quality team follow up. Markers will update results, and quality team will respond to decisions.

3. Conference Attendance Request Workflow: This allows staff to apply for conferences, and goes through an approval process in an automated way.

\section{Conclusion and Future Work}

This proposal focuses on Linkage and BIaaS 1.0. The objective is to link all different processes altogether in an integrated platform or environment. It allows different services, roles and functionalities to work together in a linkage-oriented framework. The outcome of one service can be used for another, without the need to translate from one domain or language to another. 
Advantages of BIaaS over Business Process as a Service (BPaaS) are also explained. How linkage and BIaaS works is described in a detailed case study: The University of Southampton, ECS, with its Cloud projects review, cost-saving initiatives and risk modeling. This is the BIaaS 1.0 that allows different activities to work together, and results of each stage can be used for another process. Desirable features and rationale on why BIaaS 2.0 is necessary have been explained. This will reduce level of manual extraction and computation, but also provide easy-to-use usability, enhanced security, improved collaboration and automation.

ECS, University of Southampton, has followed the CCBF first research area, Classification, for private cloud initiatives. They work for second research area, for measuring its cost-saving business performance, in which statistical computing and 3D Visualisation have been presented. The work is passed onto the third research area to compute risk modelling and analysis. The outcome of all these activities is presented as the CCBF Review and Recommendation. The University of Southampton has gained significantly with the most positive impacts as a result of BIaaS and linkage. Its actual return cost-saving is between 21.0 and $22.0 \%$, and is well above the $10 \%$ initial estimation. The combined use of Risk Analysis and Quality Assurance also allow risk control and data quality to be reviewed and monitored, and tests are used to validate our good data quality. This is a full BIaaS implementation that works towards BIaaS 2.0. Lessons learned are highly transferrable to organisations adopting Cloud. Collaboration with IBM US in BIaaS 1.0 and 2.0 development include Financial Software as a Service (FSaaS) and planned integration with IBM Fine Grained Security Model (IFGSF).

Linkage and BIaaS 1.0 have been adopted and used by organisations such as King's College London, NHS, Universities of Greenwich, Southampton and Oxford. Collaborators find it useful and contributions from Linkage and BIaaS 2.0 development will aim to positively influence different communities in Academia and Industry. The final outcome will disseminate to different communities and to help them achieve their business goals with analysis in organisational sustainability, risk modelling and enterprise portability.

\section{References}

[1] Armbrust, M., Fox, A., Griffith, R., Jseph, A. D., Katz, R. H., Kownwinski, A., Lee, G., Patterson, D., Rabkin, A., Stoica, I., Zaharia, M., "Above the Clouds: A Berkeley View of Cloud computing". Technical Report, No. UCB/EECS-2009-28, UC Berkeley, Feb 2009.

[2] Barratt, M., and Barratt, R., "Exploring internal and external supply chain linkages: Evidence from the field", Journal of Operations Management, Issue 29, p 514-528, 2011.

[3] Brandic, I., Music, D., Leitner, P. and Dustdar, S., "VieSLAF Framework: Enabling Adaptive and Versatile SLA-Management.", the 6th International Workshop on Grid Economics and Business Models 2009 (Gecon09), 25- 28 August 2009, Delft, The Netherlands.

[4] Buyya, R., Yeo, C. S., Venugopal, S., Broberg, J. and Brandic, I., "Cloud computing and emerging IT platforms: Vision, hype, and reality for delivering computing as the 5th utility", Journal of Future Generation Computer Systems, Volume 25, Issue 6, June 2009, Pages 559-616.

[5] Buyya, R., Ranjan, R. and Calheiros, R. N., "InterCloud: UtilityOriented Federation of Cloud Computing Environments for Scaling of Application Services", Algorithm and Architectures for Parallel Processing, Lecture Notes in Computer Science, 2010, Volume 6081/2010, 13-31.

[6] Chang, V., Bacigalupo, D., Wills, G., De Roure, D., "A Categorisation of Cloud Computing Business Models", poster paper, CCGrid 2010 IEEE conference, Melbourne, Australia, May 2010

[7] Chang, V., Wills, G. and De Roure, D., "A Review of Cloud Business Models and Sustainability", IEEE Cloud 2010, the third International Conference on Cloud Computing, 5-10 July 2010, Miami, Florida, USA.

[8] Chang, V., Li, C.S, De Roure, D., Wills, G., Walters, R. and Chee, C., "The Financial Clouds Review", International Journal of Cloud Applications and Computing, 1 (2). pp. 41-63, April, 2011.

[9] Chang, V., Li, C.S, De Roure, D., Wills, G., Walters, R. and Barry, T., "Cloud Computing Business Framework: Linking Operations, IT and Enterprises", submitted to Journal of Operations Management, May 2011.

[10] Chang, V., De Roure, D., Wills, G., Walters, R. and Barry, T. (2011) Organisational Sustainability Modelling for Return on Investment: Case Studies presented by a National Health Service (NHS) Trust UK. Journal of Computing and Information Technology, 19 (2). ISSN Print ISSN 1330-1136.

[11] Chang, V., De Roure, D., Wills, G. and Walters, R. (2011) "Case Studies and Organisational Sustainability Modelling presented by Cloud Computing Business Framework", International Journal of Web Services Research. ISSN 1545-7362 (In Press)

[12] Croson, R. and Donohue, K., "Impact of POS data sharing on supply chain management: an experimental study", Production and Operations Management 12 (1), 1-11, 2003.

[13] De Roure, D., Page, K.R., Fields, B., Crawford, T., Downie J. S., and Fujinaga, I., "An e-Research Approach to Web-Scale Music Analysis", in Philosophical Transactions of the Royal Society Series A, 2011.

[14] Li, C.S., "Cloud Computing in an Outcome Centric World", keynote in IEEE Cloud 2010 conference, July 5-10 2010, Miami, Florida, USA.

[15] Li, C. S., "Managing Business Integrity", Technical Report, IBM US, 2010.

[16] Pajorova E and Hluchy L, "3D visualization the results of complicated Grid and Cloud-based applications", the 14th International Conference on Intelligent Engineering Systems (INES), 5-7 May 2010, Las Palmas, Spain.

[17] Papazoglou, M.P., and Georgakopoulos, D., "Service Oriented Computing", Communications of the ACM, Vol. 46, No. 10, October 2003.

[18] Rungtusanatham, M., Salvador, F., Forza, C. and Choi, T.Y., "Supply-chain linkages and operational performance: a resourcebased perspective". International Journal of Operations and Production Management 23, 1084-1099, 2003

[19] Sharp, W.F., "Capital Asset Prices with and without Negative Holdings", Nobel-Prize Economics Lecture, December 7, 1990.

[20] Sutherland-Smith, W., and Carr, R., " Turnitin.com: Teachers' Perspectives of Anti-Plagiarism Software in Raising Issues of Educational Integrity", Journal of University Teaching and Learning Practice, 2005. 